\title{
Concurrent Squamous Cell Carcinoma and Leiomyosarcoma in the Larynx at Relapse 15 Years Post-radiation for Primary Laryngeal Cancer
}

\author{
Karolina Dorobisz ${ }^{1}$, Marcin Frączek ${ }^{1}$, Przemysław Kowalski ${ }^{2}$ and Tomasz Zatoński ${ }^{1}$ \\ ${ }^{1}$ Department of Otolaryngology, Head and Neck Surgery, Wroclaw Medical University, Borowska, Wroclaw, Poland \\ ${ }^{2}$ Department of Pathomorphology and Oncological Cytology, Wroclaw Medical University, Borowska, Wroclaw, Poland
}

\begin{abstract}
Malignant tumours of the larynx account for $50 \%$ of all head and neck neoplasms. Squamous cell carcinoma is the most common malignancy of the larynx and accounts for 95-98\%. Leiomyosarcoma (LMS) originating in larynx is a very rare malignant tumour derived from the smooth muscle tissue. The authors present a case of a 75-year man with coexistent squamous cell carcinoma and leiomyosarcoma in the larynx 15 years after radiation therapy for promany laryngeal cancer. Two cases of the synchronous presence of these neoplasms have been reported so far in English literature. This paper presents the clinical picture, radiological and histopathological diagnoses, and treatment of this case.
\end{abstract}

Key Words: Squamous cell carcinoma, Leiomyosarcoma, Larynx.

How to cite this article: Dorobisz K, Frączek M, Kowalski P, Zatoński T. Concurrent Squamous Cell Carcinoma and Leiomyosarcoma in the Larynx at Relapse 15 Years Post-radiation for Primary Laryngeal Cancer. J Coll Physicians Surg Pak 2020; 30(08):871-873.

\section{INTRODUCTION}

Malignant tumours of the larynx account for $50 \%$ of all head and neckneoplasms. Squamous cell carcinoma is the most common malignancy of thelarynxand accounts for $95-98 \% .{ }^{1}$ Leiomyosarcoma (LMS) of larynx is a very rare malignant neoplasm derived from the smooth muscle tissue, and belongs to the group of soft tissue sarcomas. It very rarely occurs in the larynx, with the incidence of less than $1 \%$ of all malignant laryngeal tumours. ${ }^{2}$ LMS located in the region of the head and neck represents only $3 \%$ of all sarcomas. The coexistence of the malignancies described above is very rare and its etiology is unclear. Two cases with synergistic squamous cell carcinoma and leiomyosarcoma in the larynx (LLMS) have been described so far in English literature.

\section{CASE REPORT}

A 75-year man was admitted to our department with recurrent sore throat, hoarseness, difficult swallowing and dyspnoea for 2 months. He was receiving regular treatment for hypertension and diabetes.

Correspondence to: Karolina Dorobisz, Department of Otolaryngology, Head and Neck Surgery, Wroclaw Medical University, Borowska, Wroclaw, Poland E-mail:dorobiszkarolina@gmail.com

Received: May 24, 2019; Revised: September 23, 2019; Accepted: October 14, 2019

DOI: https://doi.org/10.29271/jcpsp.2020.08.871
Previously, he was diagnosed with laryngeal squamous cell carcinoma 15 years ago, which was treated with radiation therapy. Since then, he was on regular check-ups and was relapse-free till his present complaints. The personal history revealed that he was a heavy smoker with history of 45 pack years. The family history was insignificant.

At admission, the indirect laryngoscopy found a tumour in the left aryepiglottic fold, true glottis, considerably narrowed subglottic area and reduced mobility of the vocal folds. The lymph nodes were impalpable. Baseline laboratory workup was unremarkable.

Computed tomography (CT) showed a lesion in the left aryepiglottic fold, with a size of 14 displaying contrast enhancement, infiltrating the upper horn of the thyroid cartilage and indenting and narrowing the lumen of the lower part of the throat. Oedematous-inflammatory lesions of the laryngeal vestibule, glottis and the subglottic area were noted. There were also enlarged lymph nodes in the left submandibular area $(7 \mathrm{~mm})$, and the left submental region ( $8 \mathrm{~mm}$ ).

The chest X-ray and abdominal ultrasound excluded the presence of distant metastases. Because of dyspnoea, the patient underwent emergency tracheotomy and the collection of material for the histopathological examination. Leiomyosarcoma was demonstrated in the histopathological examination of the lesion. Based on the clinical picture, the tumour stage was established as YCT4NOMO.

The patient was subjected to surgical treatment- total laryngectomy with left-sided radical neck disssection. 


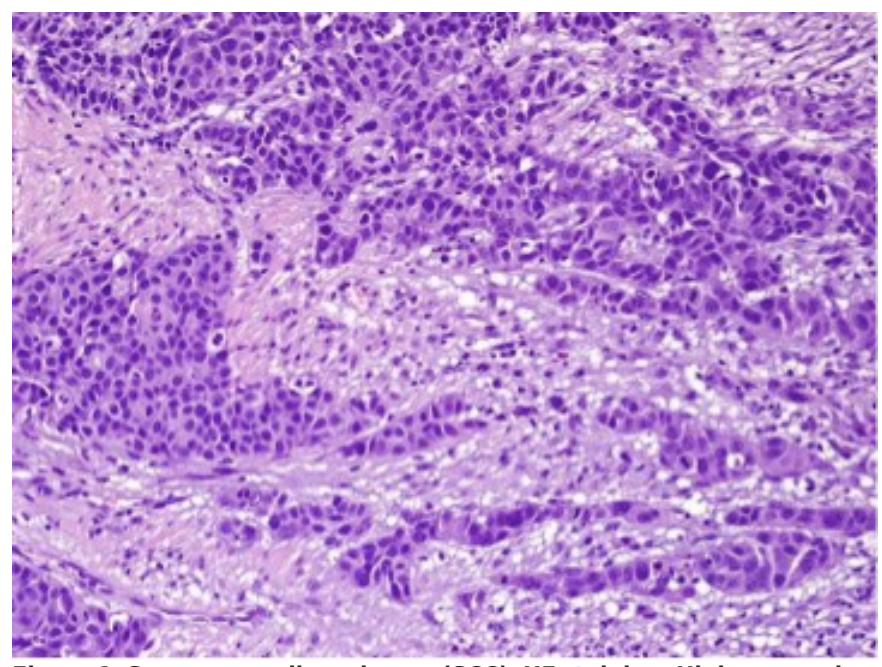

Figure 1: Squamous cell carcinoma (SCC), HE staining: High power view (original magnification 200X).

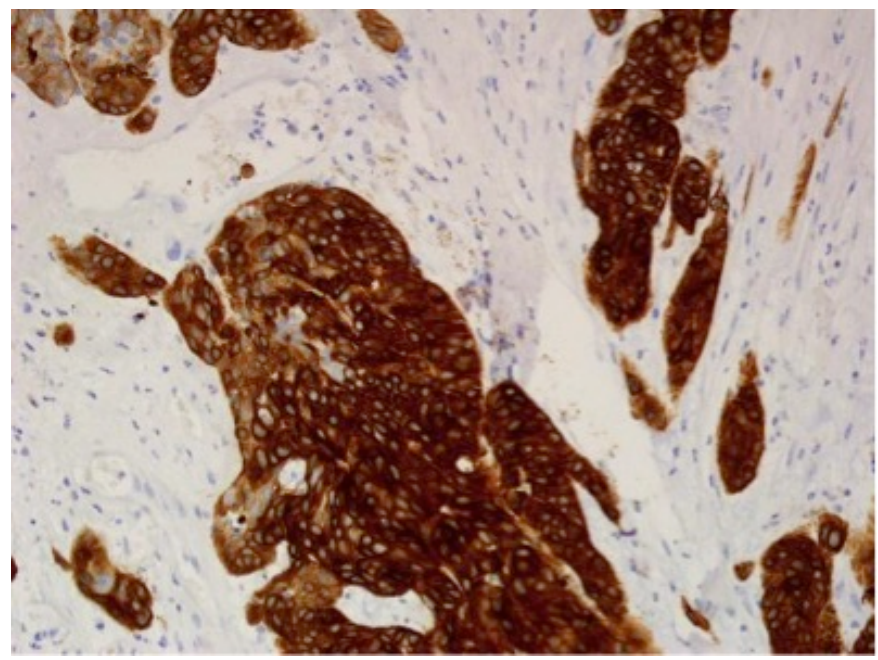

Figure 2: Squamous cell carcinoma (SCC), epithelial cells seen in reaction with cytokeratin, Ck-5/6 staining: High power view (original magnification 200X).

The histopathological result of the postoperative specimen showed moderately differentiated squamous cell carcinoma Grade 2, pathological stage ypT4NOM0. The tumour, was involving the subglottic area, the submucosal layer of the glottis on the right side and the tracheal mucosa (Figures 1 and 2). Leiomyosarcoma was found in the subglottic area (Figures 3 and 4).

The wound healed well without any complications. The patient was not offered adjuvant radiotherapy or chemotherapy. The patient is on regular surveillance in oncology outpatient clinic and is currently relapse-free.

\section{DISCUSSION}

Malignant tumours of the larynx account for $1-5 \%$ of all malignancies. Squamous cell carcinoma is the most common. ${ }^{1}$ LMS occurs very rarely in the larynx. The first case was described by Jackson in 1939. Since then, more than 50 cases have been reported in English literature. ${ }^{2,3}$ LLMS occurs in the fifth decade of life. It more often appears in men than in women, urethr ratio of $4: 1 .^{3}$ In our case, it wasa 75 -year man.

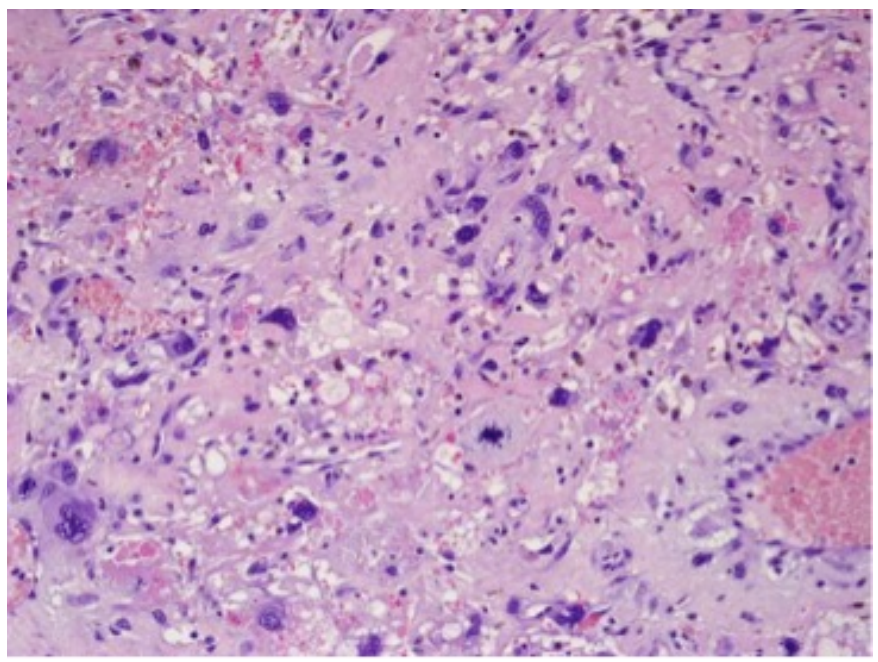

Figure 3: Leiomyosarcoma pleomorphicum, HE staining: High powerview (original magnification 200X).

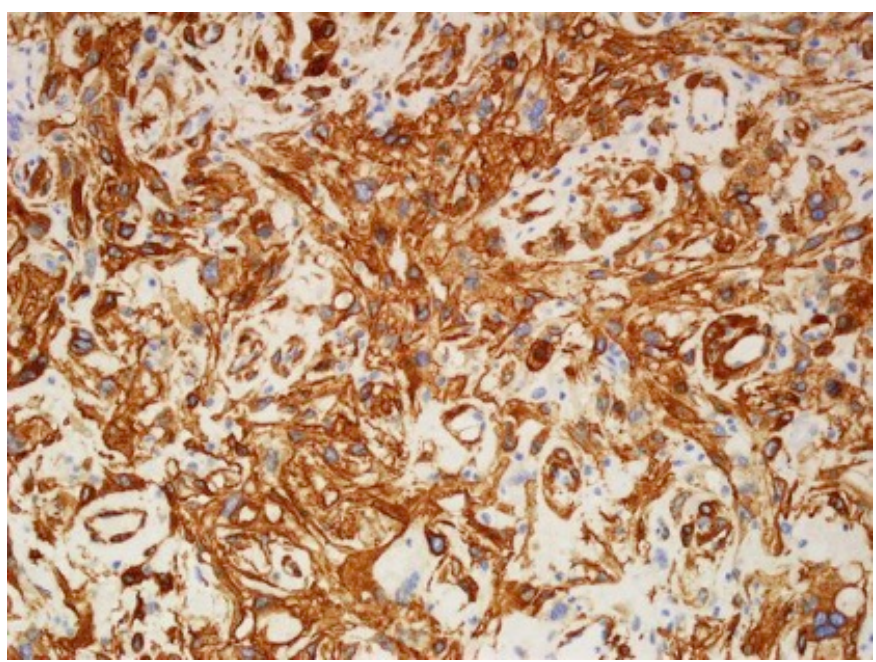

Figure 4: Leiomyosarcoma pleomorphicum, reaction seen with SMA, smooth muscle actin staining: High power view (original magnification 200X).

The coexistence of two malignant neoplasms in the larynx is extremely rare. English literature describes only 4 such cases. They included the coexistence of chondrosarcoma and squamous cell carcinoma, a denocarcinoma and squamous cell carcinoma and two cases of LMS and squamous cell carcinoma. ${ }^{4-7}$ In our case, the patient had synchronous LLMS and squamous cell carcinoma in the larynx. The etiology of malignancies of the respiratory system is unclear: Sarcoma most likely develops on the basis of mesenchymal metaplasia of the neoplastic tumour. Volker et al. suggested the development of LLMS as a local relapse of squamous cell carcinoma of the larynx. ${ }^{8}$

LLMS is usually found in the area of glottis and epiglottis. Marioni et al. have analysed 31 cases of patients with LLMS, concluding that $48 \%$ of the neoplasms involved the glottis, $32 \%$ epiglottis, and 5 to $6 \%$ the subglottic area. ${ }^{9}$ In our case, LMS was found in the subglottic region, and this is a very rare site. Apart from the larynx, LMS located in the head and neck area, can also be found in the paranasal sinuses, on the scalp or in the oesophagus.

The etiology oflaryngeal malignancies remains unclear. Forsqua- 
mous cell carcinoma, the main risk factors are smoking, alcohol consumption, and human papilloma virus (HPV) infection. However, in contrast to squamous cell carcinoma, radiotherapy, neurofibromatosis, Werner syndrome, Gardner syndrome, abnormal mesenchymal differentiation, and Epstein-Barr virus (EBV) appear to be factors predisposing to LMS. ${ }^{7}$ In our case, 15 years earlier, the patient had undergone laryngeal radiation therapy due to squamous cell carcinoma. He was a smoker; but other risk factors were not detected.

LLMS produces symptoms typical of a laryngeal tumour, such as hoarseness, dysphagia and dyspnoea: The symptoms may last from a few weeks to several years. ${ }^{1}$ In our case, the symptoms included hoarseness, throat pain, dysphagia, and recently dyspnoea. The symptoms lasted for several months. The first symptom of LLMS may be dyspnoea. Tewary et al. described a case of LLMS requiring emergency laryngectomy. ${ }^{2,10}$ Our patient required emergency tracheotomy due to dyspnoea and the large size of thetumour.

Histopathological grade of tumor and the presence of metastatic lymph nodes determine treatment and prognosis. This applies both to squamous cell carcinoma and LLMS. Both neoplasms rarely metastasize to the lymph nodes. ${ }^{2}$ In our patient, the lymph nodes were not affected. Unlike laryngeal squamous cell carcinoma, which very rarely causes distant metastases, LMS metastases usually appear later, mainly in the lungs, liver, bones, brain and skin. There were no distant metastases in this case.

Imaging studies, computed tomography and magnetic resonance imaging play a very important role in the planning of surgical treatment. Although there are no recommendations for the treatment of LLMS, surgery is the main therapeutic option in LLMS. Surgical excision with wide tumour-free margins gives good prognosis. Most surgeons choose total laryngectomy as a primary treatment, but partial laryngectomy may also be performed in case with low tumour burden. Neck dissection is not normally recommended in head and neck sarcomas. According to many authors, the treatment of coexisting LLMS and squamous cell carcinoma should be adjusted to the grade of squamous cell carcinoma. In our patient, total laryngectomy with left-sided radical neck disssection was performed due to the suspicion of lymph node metastases on the left side, as seen in the CTscan.

Radiotherapy is used in LLMS recurrence. ${ }^{2}$ Chemotherapy is rather ineffective. ${ }^{2}$ As the patient had undergone radiotherapy 15 years ago and could not be offered this modality again, only the surgical treatment was carried out.

According to literature, the prognosis of 5 -year survival in LLMS is $35-50 \%$.
LLMS is a very rare malignancy of the larynx; it extremely rarely coexists with squamous cell carcinoma. Follow-up after laryngeal cancer treatment, especially after the use of radiation therapy, should bemeticulousand regular.

\section{PATIENT'S CONSENT:}

Patient's informed consent was obtained for publication of the case data.

\section{CONFLICT OF INTEREST:}

The authors declare no conflict of interest.

\section{AUTHORS' CONTRIBUTION:}

KD, MF, PK, TZ: All authors were involved in writing the work.

KD, TZ: Carried out the research project, data acquisition, analysis and interpretation of the results.

PK: Analysed and prepared histopathological examinations.

\section{REFERENCES}

1. Skoulakis CE, Stavroulaki $P$, Moschotzopoulos $P$, Paxinos $M$, Fericean A, Valagiannis DE. Laryngeal leiomyosarcoma: A case report and review of the literature. Eur Arch Otorhinolaryngol 2006; 263(10):929-34.

2. Khadivi E, Taziky MH, Jafarian AH, Nasseri Sadr M. Laryngeal leiomyosarcoma: A case report and review of articles. Iran J Otorhinolaryngol 2013; 25(73):253-8.

3. Morera Serna E, Pérez Fernández CA, de la Fuente Jambrina C, Razquin Muñoz J, Pérez Gil MA. Laryngeal leiomyosarcoma. Acta Otorrinolaringol Esp 2007; 58:445-8.

4. Croce A, Neri G, Lattanzio G, Angelucci D. Simultaneous laryngeal carcinomas: A case report and review of literature. Acta Otorhinolaryngol Ital 1994; 14(2):157-65.

5. Obermyer NE, Ramadan HH. Adenocarcinoma with simultaneous squamous carcinomas of the larynx. Head Neck 1994; 16(5):453-6.

6. Tomidokoro Y, Hayashi R, Yamasaki M, Ishii G, Kamijo T, Ebihara S. Simultaneous squamous cell carcinoma with leiomyosarcoma of the larynx. Auris Nasus Larynx 2009; 36(2):239-43.

7. Kara E, Cetik MF, Tuncer U, Uguz A. Rare association of leiomyosarcoma with squamous cell carcinoma of the larynx: Two cases. J Laryngol Otol 2013; 127(8):828-31.

8. Völker HU, Zettl A, Haralambieva E, Blume B, Hagen R, MüllerHermelink HK et al. Leiomyosarcoma of the larynx as a local relapse of squamous cell carcinoma: Report of an unusual case. Head Neck 2010; 32(5):679-83.

9. Marioni G, Bertino G, Mariuzzi L, Bergamin-Bracale A, Lombardo M, Beltrami C. Laryngeal leiomyo-sarcoma. J Laryngol Otol 2000; 114(5):398-401.

10. Tewary AK, Pahor AL. Leiomyosarcoma of the larynx: Emergency laryngectomy. J Laryngol Otol 1991; 105(2): $134-6$. 